\title{
Strong associations and moderate predictive value of early symptoms for SARS-CoV-2 test positivity among healthcare workers, the Netherlands, March 2020
}

Alma Tostmann ${ }^{1}$, John Bradley ${ }^{2}$, Teun Bousema ${ }^{1,3}$, Wing-Kee Yiek ${ }^{1}$, Minke Holwerda $^{1}$, Chantal Bleeker-Rovers ${ }^{4}$, Jaap ten Oever ${ }^{4}$,

Corianne Meijer ${ }^{1}$, Janette Rahamat-Langendoen ${ }^{1}$, Joost Hopman ${ }^{1}$, Nannet van der Geest-Blankert ${ }^{5}$, Heiman Wertheim ${ }^{1}$

1. Department of Medical Microbiology, Radboud Centre for Infectious Diseases, Radboud university medical centre, Nijmegen, The Netherlands

2. MRC Tropical Epidemiology Group, London School of Hygiene and Tropical Medicine, London, United Kingdom

3. Department of Infection and Immunity, London School of Hygiene and Tropical Medicine, London, United Kingdom

4. Department of Internal Medicine, Radboud Centre for Infectious Diseases, Radboud university medical centre, Nijmegen, The Netherlands

5. Department of Occupational Health, Radboud university medical centre, Nijmegen, The Netherlands

Correspondence: Alma Tostmann (Alma.Tostmann@radboudumc.nl)

Citation style for this article:

Tostmann Alma, Bradley John, Bousema Teun, Yiek Wing-Kee, Holwerda Minke, Bleeker-Rovers Chantal, ten Oever Jaap , Meijer Corianne, Rahamat-Langendoen Janette, Hopman Joost, van der Geest-Blankert Nannet, Wertheim Heiman. Strong associations and moderate predictive value of early symptoms for SARS CoV-2 test positivity among healthcare workers, the Netherlands, March 2020. Euro Surveill. 2020;25(16):pii=2000508. https://doi.org/10.2807/1560-7917. ES.2020.25.16.2000508

Article submitted on 03 Apr 2020 / accepted on 21 Apr 2020 / published on 23 Apr 2020

Healthcare workers $(n=803)$ with mild symptoms were tested for severe acute respiratory syndrome coronavirus 2 (SARS-CoV-2) $(n=90$ positive) and asked to complete a symptom questionnaire. Anosmia, muscle ache, ocular pain, general malaise, headache, extreme tiredness and fever were associated with positivity. A predictive model based on these symptoms showed moderate discriminative value (sensitivity: 91.2\%; specificity: $55.6 \%$ ). While our models would not justify presumptive SARS-CoV-2 diagnosis without molecular confirmation, it can contribute to targeted screening strategies.

Following the emergence of severe acute respiratory syndrome coronavirus 2 (SARS-CoV-2) in China in December 2019, countries worldwide strive to contain or slow down virus transmission to allow health facilities to cope with the rapid rise of patients with coronavirus disease (COVID-19) [1,2]. In February 2020, the first patient with COVID-19 was reported in the Netherlands. Cases initially clustered in the province of Noord Brabant, followed by dissemination across the country [3].

Healthcare workers (HCW) are disproportionally at risk of contracting SARS-CoV-2 [4]. To protect HCW and prevent nosocomial transmission, it is recommended that healthcare facilities have a strategy for testing, management and follow-up of $\mathrm{HCW}$ with respiratory symptoms $[2,4]$. Test results also guide when an HCW can return to work with mild symptoms. Unfortunately, national and institutional strategies are in part dependent on the operational feasibility.
Here, we present our findings from a large cohort of symptomatic HCW who were tested for SARS-CoV-2 infection as part of our hospital programme. We aimed to identify symptoms associated with test positivity and develop a diagnostic model to predict SARS-CoV-2 infection based on early symptoms. In a context of limited availability of testing supplies, prediction models may support targeted testing strategies and guidelines to refrain from or resume clinical duties.

\section{Screening of healthcare workers}

Since early March 2020, HCW in our hospital have been tested for SARS-CoV-2 when they have symptoms suggestive of COVID-19 according to institutional policy based on the latest evidence. All 1,247 HCW tested between 10 and 29 March 2020 received an email with a link to an online anonymous questionnaire on the symptoms they experienced before their test. We received 803 completed questionnaires, an overall response rate of $64 \%$. By 29 March, there had been 112 $\mathrm{HCW}$ with a positive test, of whom 90 responded to the questionnaire, which suggests a slight overrepresentation of test-positives among the respondents (response rate $80 \%)$.

Initially, the questionnaire covered respiratory and general non-respiratory symptoms and was completed by $627 \mathrm{HCW}$ (56 SARS-CoV-2-positive) between 10 and 23 March 2020. After reports on anosmia and gastrointestinal illness in the initial COVID-19 patients, the questionnaire was adapted on 24 March to also include anosmia, diarrhoea, nausea and extreme tiredness. This updated questionnaire was completed by $176 \mathrm{HCW}$ 
Description of the study population of healthcare workers tested for SARS-CoV-2, by PCR result, the Netherlands, March $2020(\mathrm{n}=803)$

\begin{tabular}{|c|c|c|c|c|c|c|}
\hline & \multicolumn{2}{|c|}{$\begin{array}{l}\text { Total } \\
n=803\end{array}$} & \multicolumn{2}{|c|}{$\begin{array}{l}\text { SARS-CoV-2-positive } \\
\qquad n=90\end{array}$} & \multicolumn{2}{|c|}{$\begin{array}{l}\text { SARS-CoV-2-negative } \\
\qquad n=713\end{array}$} \\
\hline & $\mathrm{n}$ & $\%$ & $n$ & $\%$ & $\mathrm{n}$ & $\%$ \\
\hline \multicolumn{7}{|l|}{ Sex ${ }^{a}$} \\
\hline Male & 141 & 17.6 & 19 & 21.1 & 122 & 17.1 \\
\hline Female & 661 & 82.4 & 71 & 78.9 & 590 & 82.9 \\
\hline \multicolumn{7}{|l|}{ Age group (years) } \\
\hline$<20$ & 10 & 1.2 & 0 & 0.0 & 10 & 1.4 \\
\hline $21-30$ & 216 & 26.9 & 24 & 26.7 & 192 & 26.9 \\
\hline $31-40$ & 231 & 28.8 & 22 & $24 \cdot 4$ & 209 & 29.3 \\
\hline $41-50$ & 173 & 21.5 & 25 & 27.8 & 148 & 20.8 \\
\hline $51-60$ & 133 & 16.6 & 16 & 17.8 & 117 & 16.4 \\
\hline$>60$ & 40 & 5.0 & 3 & $3 \cdot 3$ & 37 & 5.2 \\
\hline \multicolumn{7}{|l|}{ Comorbidities (one or more) } \\
\hline Chronic lung disease & 61 & 7.6 & 4 & $4 \cdot 4$ & 57 & 8.0 \\
\hline Disease of immune system & 29 & 3.6 & 0 & 0.0 & 29 & 4.1 \\
\hline Allergy & 137 & 17.1 & 8 & 8.9 & 129 & 18.1 \\
\hline \multicolumn{7}{|l|}{ Medical profession } \\
\hline Medical doctor & 144 & 17.9 & 21 & 23.3 & 123 & $17 \cdot 3$ \\
\hline Nurse & 266 & 33.1 & 31 & 34.4 & 235 & 33.0 \\
\hline Other healthcare worker & 393 & 48.9 & 38 & 42.2 & 355 & 49.8 \\
\hline \multicolumn{7}{|l|}{ Questionnaire } \\
\hline Initial questionnaire (for development of model) & 627 & 78.1 & 56 & 62.2 & 571 & 80.1 \\
\hline Extended questionnaire (for validation of the model) & 176 & 21.9 & 34 & 37.8 & 142 & $19 \cdot 9$ \\
\hline
\end{tabular}

SARS-CoV-2: severe acute respiratory syndrome coronavirus 2.

${ }^{a}$ For one person information on sex was missing.

(34 positives) between 24 and 30 March 2020. Most respondents were female (82.9\%) and between 21 and 40 years-old $(55.7 \%)$ (Table 1$)$.

To allow a diagnostic model to be created based on the initial cohort $(627 \mathrm{HCW})$ and tested in the second cohort (176 HCW), all HCW who were tested positive before 24 March $(n=77)$ and a random selection ( $n=$ 99) of the HCW who tested negative before 24 March received an additional questionnaire asking about these four additional symptoms separately. This additional questionnaire was completed by 45 SARS-CoV-2positive and 48 negative HCW.

\section{Symptoms associated with SARS-CoV-2- positive test results}

The most frequently reported symptoms among testnegative HCW were cough $(60 \%)$, sore throat (56\%) and common cold (51\%). Test-positive HCW most frequently reported headache $(71 \%)$, general malaise (63\%) and muscle ache (63\%) (Table 2). Among 176 $\mathrm{HCW}$ who responded to the second questionnaire after 24 March, HCW who tested positive for SARS-CoV-2 had a median of four symptoms (interquartile range
(IQR): 2-6) compared with three symptoms (IQR: 2-5) for HCW who tested negative $(p=0.004)$.

Univariate associations were assessed by calculating odds ratios. General non-respiratory symptoms (muscle ache, ocular pain, general malaise, headache and extreme tiredness) were associated with test positivity. Anosmia was reported by $47 \%$ of test-positives and was strongly associated with SARS-CoV-2 positivity $(\mathrm{OR}=23.0$; 95\% confidence interval $(\mathrm{Cl}): 8.2-64.8)$. Among the 14 people with anosmia in whom other symptoms were also recorded, 10 reported a runny nose and/or sneezing, while four did not report any symptoms that can cause nasal congestion. Among 10 individuals with both anosmia and muscle ache, nine were SARS-CoV-2-positive. None of the respiratory symptoms were associated with SARS-CoV-2 positivity, sore throat being less common among test positives (40.0\% vs 56.1\%; $p=0.004)$. Symptoms reported by test-positive HCW are presented in a heat map indicating which symptoms were reported together (Figure 1).

Data from the initial cohort $(627 \mathrm{HCW})$ were used to predict the SARS-CoV-2 test result in the second cohort $(176 \mathrm{HCW})$. A prediction model was fit on all 
TABLE 2

Univariate associations of early symptoms with SARS-CoV2 PCR positivity among healthcare workers, the Netherlands, March $2020(\mathrm{n}=803)$

\begin{tabular}{|c|c|c|c|c|c|c|}
\hline \multirow[t]{2}{*}{ Symptom } & \multicolumn{2}{|c|}{ SARS-CoV2-positive } & \multicolumn{2}{|c|}{ SARS-CoV2-negative } & \multirow[t]{2}{*}{ OR $(95 \% \mathrm{Cl})$} & \multirow{2}{*}{ p value } \\
\hline & $\mathrm{n} / \mathrm{N}$ & $\%$ & $\mathrm{n} / \mathrm{N}$ & $\%$ & & \\
\hline \multicolumn{7}{|c|}{ General non-respiratory symptoms } \\
\hline Anosmia $^{\mathrm{a}}$ & $37 / 79$ & 46.8 & $7 / 190$ & 3.7 & $23.0(8.2-64.8)$ & $<0.001$ \\
\hline Muscle ache & $57 / 90$ & $63 \cdot 3$ & $143 / 713$ & 20.1 & $6.9(4.2-11.3)$ & $<0.001$ \\
\hline Ocular pain & $31 / 90$ & $34 \cdot 4$ & $75 / 713$ & 10.5 & $4.5(2.7-7.4)$ & $<0.001$ \\
\hline General malaise & $57 / 90$ & 63.3 & $208 / 713$ & 29.2 & $4.2(2.6-6.7)$ & $<0.001$ \\
\hline Headache & $64 / 90$ & 71.1 & $296 / 713$ & 41.5 & $3.5(2.1-5.7)$ & $<0.001$ \\
\hline Extreme tiredness $^{a}$ & $45 / 79$ & 57.0 & $61 / 190$ & 32.1 & $2.8(1.6-4.9)$ & $<0.001$ \\
\hline Fever & $51 / 90$ & 56.7 & $233 / 713$ & 32.7 & $2.7(1.7-4.2)$ & $<0.001$ \\
\hline \multicolumn{7}{|c|}{ Respiratory symptoms } \\
\hline Common cold & $50 / 90$ & 55.6 & $363 / 713$ & 50.9 & $1.2(0.8-1.9)$ & 0.406 \\
\hline Sneeze & $36 / 90$ & 40.0 & $253 / 713$ & $35 \cdot 5$ & $1.2(0.8-1.9)$ & 0.401 \\
\hline Cough & $53 / 90$ & 58.9 & $424 / 713$ & $59 \cdot 5$ & $1.0(0.6-1.5)$ & 0.916 \\
\hline Shortness of breath & $20 / 90$ & 22.2 & $157 / 713$ & 22.0 & $1.0(0.6-1.7)$ & 0.965 \\
\hline Runny nose & $24 / 90$ & 26.7 & $231 / 713$ & 32.4 & $0.8\left(0.5^{-1.2}\right)$ & 0.271 \\
\hline Sore throat & $36 / 90$ & 40.0 & $400 / 713$ & 56.1 & $0.5(0.3-0.8)$ & 0.004 \\
\hline \multicolumn{7}{|c|}{ Gastrointestinal symptoms } \\
\hline Nausea $^{a}$ & $13 / 79$ & 16.5 & $17 / 190$ & 8.9 & $2.0(0.9-4.4)$ & 0.075 \\
\hline Diarrhoea $^{a}$ & $14 / 79$ & 17.7 & $20 / 190$ & 10.5 & $1.8(0.9-3.9)$ & 0.106 \\
\hline
\end{tabular}

SARS-CoV-2: severe acute respiratory syndrome coronavirus 2.

${ }^{a}$ The total here includes only those respondents of the second response cohort ( 34 cases and 142 non-cases) and those from the first cohort who responded to the additional questions ( 45 cases and 48 non-cases).

symptoms using Lasso regression (STATA version 16.0; Statacorp, Texas, United States) [5]. This is a technique that attempts to reduce overfitting by shrinking coefficients. Some coefficients are shrunk to zero and hence effectively removed from the model. The model retained all variables except fever and cough. This model achieved an area under the ROC (receiver operating characteristic) curve of 0.754 (95\% Cl: $0.662-0.846$ ) (Figure $2 \mathrm{~A}$ ) and achieved a sensitivity of $82.4 \%$ and specificity of $59.2 \%$. A simple model which included all the symptoms with a significant positive association, weighted based on the results in Table 2 (weight of 3 for anosmia, 2 for muscle ache and 1 each for extreme tiredness, headache, ocular pain fever, and general malaise) achieved an area under the ROC curve of 0.783 (95\% Cl: $0.696-0.870)$ (Figure 2B). Using a cut-off of $\geq 3$ symptoms gave a sensitivity of $91.2 \%$ and a specificity of $55.6 \%$ for SARS-CoV-2 test positivity.

\section{Discussion}

This study provides valuable insights in the early symptoms of COVID-19 in a large cohort of HCW. General non-respiratory symptoms (muscle ache, ocular pain, general malaise, headache, extreme tiredness and fever) were most frequently reported by test-positive HCW and these symptoms were strongly associated with SARS-CoV-2 test positivity, unlike respiratory symptoms such as cough and sneezing. Anosmia was particularly strongly associated with test positivity.
Fever and cough have been reported as early symptoms in mild COVID-19 cases [6], and fever occurs in the majority of individuals hospitalised for COVID-19 [7]. However, in our study fever was not the strongest predictor of test positivity among $\mathrm{HCW}$, which is in line with findings from a similar study from the Netherlands [8].

Recent work from the Netherlands showed that $63 \%$ of HCW had worked despite mild symptoms [8]. Clear guidance on the use of personal protective equipment (PPE) and clear protocols for work abstinence during symptoms are vital to protect $\mathrm{HCW}$ and patients. Models of triage and diagnosis may help physicians and public health authorities to estimate infection risk and support decisions about such isolation and quarantine measures [9]. Our simple prediction model based on the seven symptoms most strongly associated with SARS-CoV-2 positivity among HCW, giving extra weight to anosmia and muscle ache as strongest predictors, gave a high sensitivity (91.2\%) and a moderate specificity (55.6\%). Where the sensitivity and specificity of a diagnostic test or prediction algorithm are features of the test, the positive predictive value (PPV) and negative predictive value (NPV) are related to the infection prevalence in the population. With increasing prevalence, the PPV increases and NPV decreases. With a prevalence of infection of $10 \%$, the above sensitivity and specificity would result in a PPV 


\section{FIGURE 1}

Heatmap of early symptoms reported by healthcare workers positive for SARS-CoV-2, the Netherlands, March $2020(\mathrm{n}=90)$

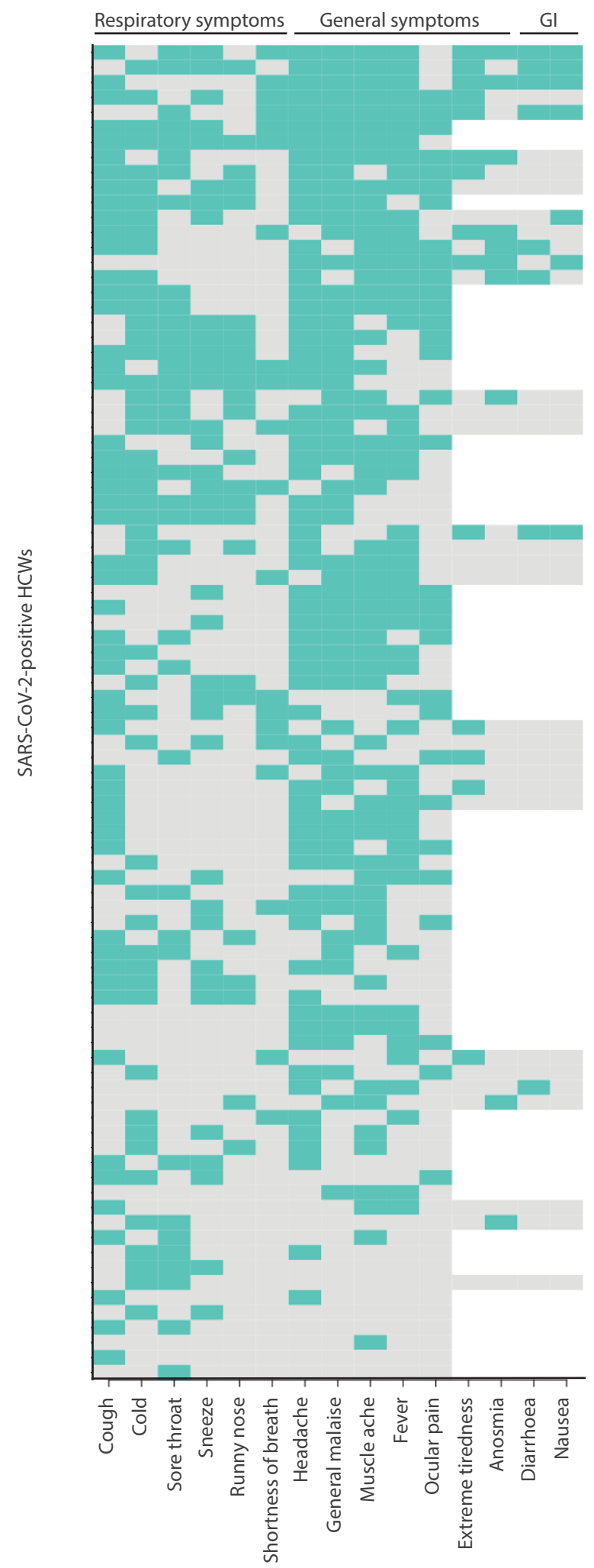

GI: gastrointestinal; SARS-CoV-2: severe acute respiratory syndrome coronavirus 2 . of $16.9 \%$ and NPV of $98.5 \%$. This is very informative, as with only $1.5 \%$ chance of having SARS-CoV-2 upon a negative 'prediction', hospitals could consider letting those HCW work (with PPE), depending on the shortage of staff. Because of the low specificity, about half of HCW would be predicted as 'positive'. However, testing only this subgroup of 'predicted positives' would reduce the necessary testing materials by $50 \%$.

A unique aspect of our study is that we assessed early symptoms of COVID-19 in an otherwise healthy population. The $\mathrm{HCW}$ received the invitation to the questionnaire the day after receiving their test result, which was usually within $24 \mathrm{~h}$ after the sample was taken, thus minimising recall bias. The response rate was $64 \%$, and selection bias could have occurred as people with atypical (i.e. non-respiratory symptoms) could have been more likely to respond to the questionnaire, leading to an overrepresentation of people with these symptoms. However, as the percentage of respiratory symptoms among test-positive and test-negative HCW was comparable, we do not expect that this could have explained the strong associations observed in this study. Our anonymous data collection did not allow us to examine initial symptoms in relation to disease progression. The study design would have allowed for the calculation of relative risks (RR), were it not that we had asked for four symptoms in a case-control manner. Even though RR are easier to interpret than $O R$, and $O R$ may slightly overestimate the risk estimate, presenting a mix of RR and OR would have been confusing. In addition, presenting the $O R$ with column percentages gives the useful insight of the percentage of cases (and noncases) that reported a certain symptoms.

\section{Conclusion}

Our findings may be used to refine national public health guidelines on self-isolating individuals whose symptoms are suggestive of COVID-19. The current policy in the Netherlands is that people with mild respiratory symptoms self-isolate and household members of people with fever are requested to self-quarantine. Based on our findings, this could be expanded with general non-respiratory symptoms or anosmia. Our detailed report of early symptoms among HCW tested for SARS-CoV-2 identified that general non-respiratory symptoms and anosmia were strongly associated with test positivity. While our prediction models would not justify presumptive SARS-CoV-2 diagnosis without molecular confirmation, findings may contribute to a targeted screening strategy which may be of value in settings with limited availability of testing materials. 


\section{FIGURE 2}

ROC curves predicting SARS-CoV-2 test results of healthcare workers (cohort $2 ; \mathrm{n}=176$ ) based on reported symptoms in cohort $1(\mathrm{n}=627)$, the Netherlands, March 2020

\section{A. Lasso regression using all symptoms}

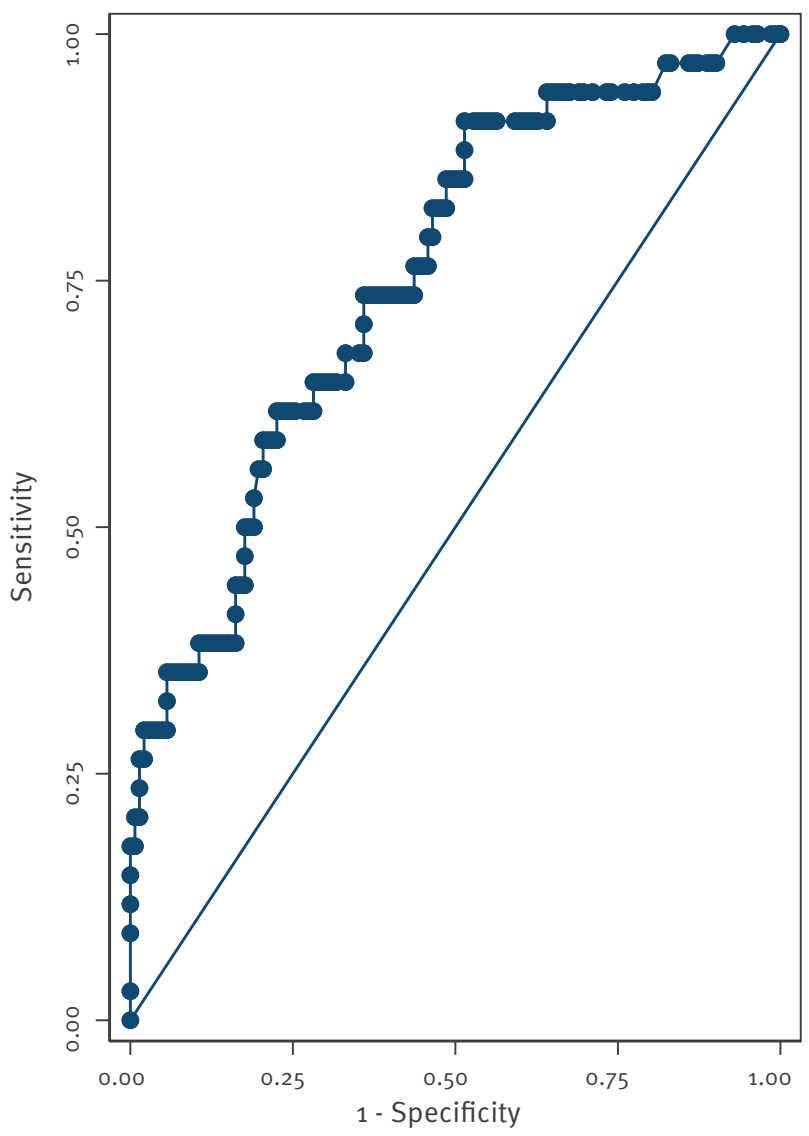

Area under ROC curve $=0.7548$

\section{B. Weighted association}

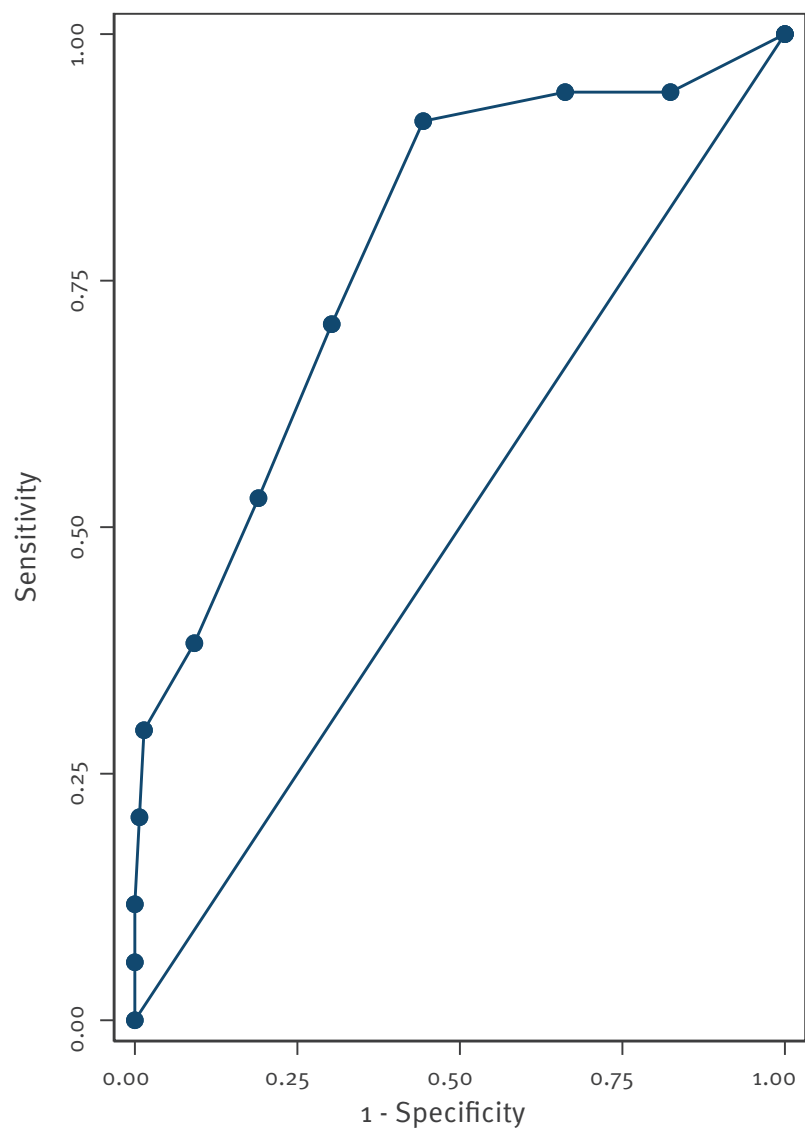

Area under ROC curve $=0.7831$

ROC curve: receiver operating characteristic curve; SARS-CoV-2: severe acute respiratory syndrome coronavirus 2.

Weighting applied in panel B: 3 for anosmia, 2 for muscle ache and 1 each for extreme tiredness, headache, ocular pain, fever and general malaise.

\section{Acknowledgements}

This work was conducted on behalf of het Radboudumc Center for Infectious Diseases COVID-19 Team. We thank Lisette Meerstein-Kessel for the production of the heat map.

Funding: JB received support from the UK MRC and the UK DFID (\#MR/Ko12126/1) under the MRC/DFID Concordat agreement and as part of the EDCTP 2 programme supported by the European Union.

\section{Conflict of interest}

None declared.

\section{Authors' contributions}

AT conceived and coordinated the study. WY and MH created the questionnaire and performed the data collection. JB and
TB conducted the data analysis and the prediction modelling. $C B, J t O, C M, J R, J H, N v d G$ and $H W$ are members of the Radboud RCI COVID-19 team, they have been involved in the development of our institutional policy for HCW screening and gave input in the study design. All authors have commented on the draft and approved the final version.

\section{References}

1. World Health Organization (WHO). Coronavirus disease (COVID-19) situation report 74. Geneva WHO; 3 Apr 2020. Available from: https://www.who.int/docs/default-source/ coronaviruse/situation-reports/20200403-sitrep-74-covid-19mp.pdf?sfvrsn=4e043d03_14

2. European Centre for Disease Prevention and Control (ECDC). Rapid risk assessment: Coronavirus disease 2019 (COVID-19) pandemic: increased transmission in the EU/EEA and the UK - seventh update. Stockholm: ECDC; 25 Mar 2020. Available from: https://www.ecdc.europa.eu/en/publications-data/ rapid-risk-assessment-coronavirus-disease-2019-covid-19pandemic 
3. National Institute for Public Health and the Environment (RIVM). Current information about the novel

coronavirus (COVID-19) Bilthoven: RIVM. [Accessed:

4 Apr 2020]. Available from: https://www.rivm.nl/en/ current-information-about-novel-coronavirus-covid-19

4. European Centre for Disease Prevention and Control (ECDC). Infection prevention and control for COVID-19 in healthcare settings - second update, 31 March 2020. Stockholm: ECDC; 2020. Available from: https://www.ecdc.europa.eu/sites/ default/files/documents/Infection-prevention-control-for-thecare-of-patients-with-2019-nCoV-healthcare-settings_update31-March-2020.pdf

5. Tibshirani R. Regression shrinkage and selection via the lasso. J R Stat Soc B. 1996;58(1):267-88. https://doi. org/10.1111/j.2517-6161.1996.tbo2080.x

6. Wei WE, Li Z, Chiew CJ, Yong SE, Toh MP, Lee VJ. Presymptomatic Transmission of SARS-CoV-2 - Singapore, January 23-March 16, 2020. MMWR Morb Mortal Wkly Rep. 2020;69(14):411-5. https://doi.org/10.15585/mmwr.mm6914e1 PMID: 32271722

7. Hu Z, Song C, Xu C, Jin G, Chen Y, Xu X, et al. Clinical characteristics of 24 asymptomatic infections with COVID-19 screened among close contacts in Nanjing, China. Sci China Life Sci. 2020. [Epub ahead of print]. https://doi.org/10.1007/ S11427-020-1661-4 PMID: 32146694

8. Kluytmans $M$, Buiting $A$, Pas $S$, Bentvelsen $R$, van den Bijllaardt W, van Oudheusden A, et al. SARS-CoV-2 infection in 86 healthcare workers in two Dutch hospitals in March 2020. medRxiv. 2020:2020.03.23.20041913.

9. Leung GM, Hedley AJ, Kong J, Lam TH, Lau FL, Rainer T, et al. A clinical prediction rule for diagnosing severe acute respiratory syndrome in the emergency department. Hong Kong Med J. 2008;14(5) Suppl;8-10. PMID: 18941266

\section{License, supplementary material and copyright}

This is an open-access article distributed under the terms of the Creative Commons Attribution (CC BY 4.0) Licence. You may share and adapt the material, but must give appropriate credit to the source, provide a link to the licence and indicate if changes were made.

Any supplementary material referenced in the article can be found in the online version.

This article is copyright of the authors or their affiliated institutions, 2020. 